\title{
De la verve. À la recherche d'un idéal discursif ordinaire
}

Jean-Claude Beacco

\section{(2) OpenEdition}

12 Journals

Édition électronique

URL : http://journals.openedition.org/pratiques/1214

DOI : $10.4000 /$ pratiques. 1214

ISSN : 2425-2042

Éditeur

Centre de recherche sur les médiations (CREM)

\section{Édition imprimée}

Date de publication : 15 décembre 2008

Pagination : 129-147

\section{Référence électronique}

Jean-Claude Beacco, «De la verve. À la recherche d'un idéal discursif ordinaire », Pratiques [En ligne], 139-140 | 2008, mis en ligne le 15 décembre 2008, consulté le 08 mars 2021. URL : http:// journals.openedition.org/pratiques/1214; DOI : https://doi.org/10.4000/pratiques.1214

(c) Tous droits réservés 


\title{
De la verve. À la recherche d'un idéal discursif ordinaire
}

\author{
Jean-Claude Beacco \\ Université Paris III-Sorbonne nouvelle \\ EA 2288 : Didactique des langues, des textes \\ et des cultures (DILTEC)
}

\begin{abstract}
Nous nous proposons d'analyser un aspect particulier de la « linguistique populaire », à savoir les conceptions circulantes de ce que sont le bien parler ou le bien écrire authentiques, qui répondent à des valeurs distinctes de ou opposées à celles qui fondent les normes dominantes dites, indifféremment, académiques, officielles, scolaires, surveillées, élégantes... Mais analyser des croyances, des savoirs ou des pratiques assignés à la " linguistique populaire » suppose, de manière liminaire, d'avoir circonscrit le champ de celle-ci. Nous commencerons donc par nous situer par rapport à ce domaine, assez peu exploré dans l'espace francophone, de la sociolinguistique.
\end{abstract}

\section{Formes des savoirs ordinaires sur le langage, les langues et les pratiques langagières}

On sait que la nature de l'étendue de la linguistique populaire et que le tracé de ses frontières incertaines sont à reconduire à l'interprétation que l'on donne de $p o^{-}$ pulaire et, en conséquence, au statut épistémologique attribuable à ces savoirs. Pour notre part, dans la continuité du numéro de Langages (Beacco 2004) consacré à ces problématiques, nous continuerons à privilégier le terme d'ordinaire, qui évite les connotations péjoratives de l'adjectif populaire, et qui s'inscrit dans une tradition bien ancrée dans le domaine sociolinguistique français (Gadet 1989 et 2003). Mais ce choix terminologique n'apporte pas d'éclairage particulier sur la nature de ces savoirs.

\subsection{Représentations sociales}

Nous utiliserons, à la suite de beaucoup d'autres, le concept de représentation sociale, pour renvoyer à certains de ces savoirs ordinaires. Dans la définition, devenue classique, de D. Jodelet (1989 p. 36), une représentation sociale est « une forme de connaissance, socialement élaborée et partagée, ayant une visée pratique et concourant à la construction d'une réalité commune à un ensemble social ». Cette spécifica- 
tion souligne le caractère diffus de ces savoirs (partagée, réalité commune à un ensemble social) et instaure donc ceux-ci en horizons ordinaires de la connaissance, mais elle souligne aussi la complexité de leur genèse (socialement élaborée), qui semble exclure une relation immédiate avec l'expérience personnelle : au contraire, elle renvoie aux stratégies que les acteurs sociaux mettent en œuvre pour sélectionner des informations socialement efficaces et, en principe, compatibles avec celles qu'ils utilisent déjà. Ces savoirs sont en partie préformés, transmis ou diffusés comme tels par toutes les voies de la communication sociale (dont l'école, les médias, la transmission familiale ou de proximité, etc.), qui peut les modifier. Ils prennent facilement des formes de stéréotypes, formules discursives compactes et fortement assertées (sous forme de définition ou de règle et d'exception à la règle, par exemple), à valeur générique, ce qui en assure à la fois la mémorisation et l'efficacité argumentative.

B. Py (2004 p. 8) en propose la formulation suivante qui décrit très heureusement les aspects que nous venons à peine de souligner :

« une représentation sociale est pour nous une microthéorie prête à l'emploi. C'est une microthéorie "économique" en ce sens qu'elle réunit en elle une grande simplicité et un vaste domaine d'application. [...] Le caractère préfabriqué des formules qui véhiculent le plus souvent les représentations sociales participe à cet aspect économique : elles sont immédiatement disponibles, elles ne requièrent aucun travail autre que l'acte de leur mise en œuvre énonciative. Leur diffusion leur confère une apparente légitimité, elles se donnent pour des constats factuels avérés plus que pour des positions théoriques destinées à un débat argumenté [...] Ces microthéories ont pour fonction de fournir (souvent dans l'urgence) des interprétations utiles à une activité en cours, qu'il s'agisse d'activité technique (prendre une décision au cours d'une action) ou symbolique (argumenter dans le cadre d'une discussion)».

Les représentations sociales peuvent ainsi concerner le langage, les langues particulières ou la communication verbale. Dans ce cas, elles mettent en jeu ce que l'on a pris l'habitude de désigner sous le terme d' " attitudes linguistiques », qui se manifestent sous la forme de croyances et de jugements de valeurs. Cet ensemble d'évaluations « héritées » et adoptées est accroché à des valeurs (bien parler en public, bien prononcer, être poli, avoir un bon accent...). Dans ce cas, ces évaluations ne sont pas à considérer sous l'angle du vrai ou du faux scientifiques mais à décrire et à expliciter en fonction des locuteurs et des groupes avec lesquels ils ont établi des relations d'appartenance privilégiée ou exclusive. Cette perception socialement filtrée des réalités linguistiques diverge nécessairement des points de vue des linguistiques professionnels, qui n'ont pas recours à des jugements de valeur sur les langues et leurs pratiques comme catégories descriptives.

Mais les représentations sociales des langues sont aussi potentiellement divergentes entre elles, car elles sont « communes à un ensemble social » (Jodelet, op. cit.), dont la nature peut être éminemment variable en fonction des critères sociologiques habituels : âge, sexe, capital scolaire, résidence urbaine / périurbaine, langue(s) identitaire(s), revenus, degré de reconnaissance sociale... On ne peut exclure l'hypothèse que certaines soient transversales à de nombreux groupes sociaux, professionnels ou culturels et non actives uniquement chez les spécialistes des langues. Ces représentations sociales « diffuses », celles « des gens », du "general public" et ses "lay comments" (1) (Gester 2001), ne sont pas aisées à identifier, car elles supposent le traitement de données statistiquement représentatives.

(1) Le grand public et ses commentaires profanes (notre traduction) 


\subsection{Références partagées}

Dans la conception courante des représentations sociales, on pose que les acteurs sociaux adhérent à celles-ci et que cette adhésion se manifeste à des actions et des comportements sociaux concrets et quantifiables. Croire qu'être mince, c'est être beau / belle devrait conduire à des comportements alimentaires ou de consommateur caractérisés. Or, dans le cas des croyances linguistiques, ces effets des représentations sur les comportements langagiers ne sont pas faciles à appréhender et encore moins à mesurer, car ils impliquent des observations longitudinales lourdes. Mais surtout l'adéquation entre valeurs / croyances et comportements langagiers n'est pas de nature déterministe et elle peut même ne pas exister : croire et dire que « le français est en déclin » n'empêche pas l'usage oral systématique de la négation pas (au lieu de ne... pas). Les représentations sociales sont des objets discursifs « mis en circulation » dans les interactions verbales. Or, c'est une banalité épistémologique de souligner que les discours ne peuvent pas être tenus pour des indices fiables des comportements effectifs, lesquels peuvent d'ailleurs échapper aux acteurs sociaux eux-mêmes. C. Oger et C. Ollivier-Yaniv (2007 p. 51) notent qu'il serait vain «à travers les entretiens, de prétendre repérer quelles représentations les acteurs se font de leur activité, tout au plus pourra-t-on déterminer quelle représentation ils en donnent - volontairement ou non - dans le contexte de l'interaction avec le chercheur ». B. Py (2004 p. 10) manifeste autrement la même réserve, d'orientation ethnométhodologique, quand il distingue

«l'accès et l'adhésion personnelle à une représentation sociale. La communication au sein d'une communauté culturelle présuppose l'accès à un répertoire de représentations sociales (accès qui permet une interprétation relativement univoque de certains énoncés), mais pas nécessairement une adhésion à ces mêmes représentations sociales. En d'autres termes, une même représentation sociale peut exprimer une conviction, voire servir de maxime de comportement, ou plus modestement de simple référence ou de convention utile ou même nécessaire à l'interprétation de certains énoncés ou comportements. Nous utiliserons respectivement les termes de représentation sociale d'usage et représentation sociale de référence ».

Or la recherche dans ce domaine se fonde surtout sur des analyses d'entretiens qui ne permettent pas de savoir si les représentations des langues préexistent à l'entretien et y émergent ou si elles prennent corps dans ces entretiens.

\subsection{Métalangage interne ou autoreprésentation des langues}

À côté des croyances et valeurs, plus ou moins ancrées chez les locuteurs et présentes dans leurs discours, il existe une troisième catégorie de savoirs sur les langues qui peut être sollicitée dans les discours mais qui proviennent de la langue ellemême. On veut désigner par là un ensemble complexe de «mots de la langue » qui constituent un métalangage non savant, au sens où il n'est pas utilisé comme tel par les linguistes professionnels, mais disponible pour décrire les pratiques langagières.

Ainsi, l'on peut s'accorder sur la définition linguistique à donner à acte de langage et intention de communication, notions d'ailleurs en germe dans l'opposition établie en français entre « ce que l'on dit» et « ce que l'on veut dire ». Mais une dénomination univoque des actes de langage dans une langue donnée (et a fortiori d'une langue à l'autre) est problématique, car le linguiste est prisonnier des termes disponibles dans le lexique. Preuve en est l'emploi laxiste qui est fait de cette catégorie 
dans les manuels d'enseignement du français comme langue étrangère (ou téléphoner et se repérer dans le temps sont donnés comme actes de langage), ou la multiplicité des dénominations disponibles, encombrante dès qu'il s'agit de les nommer : doit-on distinguer deux actes de langage puisqu'il existe en français (au moins) deux verbes proches, expliquer et expliciter? De même pour décrire et représenter, exprimer la tristesse ou l'amertume. Peut-on identifier un comportement pragmatique actualisé par des moyens linguistiques stables et suffisamment codés/prévisibles qui répondrait à l'acte exprimer le fait de ne pas être surpris, qui ferait pendant à exprimer sa surprise. On pourrait examiner de près les différences dans les typologies de ces mêmes actes qui ont une certaine vocation à l' exhaustivité, en particulier, celles proposées par des référentiels comme Un niveau seuil (Martins-Baltar 1976) et celles retenues dans Niveau B2 pour le français (Beacco et autres 2004). Et 1'on connaît les tentatives de A. Wiersbicka (1985) de décrire les actes de langage identifiés dans différentes langues au moyen de catégories universelles et donc linguistiquement et culturellement neutres.

Il en va de même pour les dénominations des genres discursifs, à ceci près que leur statut «d'objet linguistique naturel » a été théorisé par D. Hymes et M. Bakhtine, entre autres. Si les genres discursifs constituent la forme sous laquelle une langue donne prise immédiate aux locuteurs, ceux-ci sont capables de participer à l'énonciation de certains d'entre eux, en fonction de leur répertoire discursif; ils sont aussi capables, de ce fait, de les identifier, de les reconnaître et de les nommer.

«Ces représentations ordinaires des genres sont inscrites dans le lexique de la langue mais relèvent aussi de descriptions linguistiques "savantes", en tant qu'un genre discursif est une forme structurant la communication sociale, distincte de l'énoncé, du texte... » (Beacco et Petit 2004, p. 87).

Mais le fait que des noms soient disponibles pour identifier des formes discursives n'en garantit pas «l'existence». Par existence on entend la possibilité de les caractériser en fonction des catégories communément utilisées en analyse du discours, comme les conditions de production, de circulation et de réception, comme les constantes de représentation (Beacco 1982 ; au sens de présence / absence concordante d'opérations énonciatives, d'actes de langage, de type de chaîne anaphorique... et de choix dominants parmi les marqueurs disponibles en langue pour chacun de ces éléments) ou comme les constantes de cohérence, qui permettraient de mettre en évidence des formes de dispositio propres à un genre discursif. Est-ce qu'un savon (dans prendre un savon) est distinct d'une engueulade et une dispute d'une explication, prises toutes deux au sens de querelle?

Ces noms d'actes de langage et de genres discursifs ne constituent qu'une faible partie des moyens lexicaux qui se rapportent à la parole et qui sont rendus disponibles dans la langue elle-même, telle qu'elle est enregistrée dans les dictionnaires. R. Vivès (1998 et 2004) a montré que ces « verbes du dire » proposent des représentations sémantiques multiples et très hétérogènes de l'activité verbale, qui vont de bourrer le crâne à quelqu'un et se crêper le chignon à murmurer, prétendre, soupirer (que) ou encore couper la parole, crier sur les toits...

Il n'est pas aisé de caractériser la nature de ces « savoirs descriptifs » d'une langue, d'autant qu'ils ne sont connus que par le truchement des lexicographes qui introduisent une médiation de plus entre les «créateurs » de ces lexèmes et ses analystes. S'il est clair que, comme tout mot d'une langue, ils répondent à une certaine fonctionnalité de la communication (qu'elle soit de nature pratique, affective, symbolique...), on en peut rien avancer sur leurs origines sinon qu'elles ne sont pas plus 
populaires ou ordinaires que d'autres vocables. Quant à leurs emplois définis en termes sociolinguistiques, ils sont largement méconnus sauf à travers la notion très sollicitée en lexicographie, de registre, dont on sait ce qu'il faut penser en termes de présupposés normatifs. Ce sous-ensemble du lexique semble surtout représenter intuitivement des événements de communication mettant en jeu des interactions quotidiennes, perçues des points de vue qu'en donne l'expérience directe, à savoir : les circonstances de communication qui constituent ces événements, leur objet, leurs contenus ou leur finalité sociale globale, leur ton (comme ethos) et leurs qualités orales / vocales (dont l'intensité).

Ce métalangage non cohérent et non savant (en ce sens qu'il n'est pas utilisé dans les descriptions linguistiques) constitue une autoreprésentation de la langue. Du fait de sa nature, celle-ci me semble devoir être traitée dans un cadre théorique différent de celui que l'on peut construire pour l'analyse des représentations sociales des langues et de leurs usages, telle qu'elles circulent ou sont convoquées dans les interactions.

\subsection{Circulations des savoirs sur les langues}

On peut ainsi admettre que les savoirs sur les langues sont de nature cognitive différenciée : représentations sociales auxquelles on adhère, références partagées, c'est-à-dire connaissances mentionnées dans 1'intertexte discursif, et autoreprésentations, hors contexte dont il conviendrait surtout d'observer les emplois. Ces trois formes de connaissance métalinguistique sont conjointement à la disposition des acteurs sociaux Mais il faut articuler ces connaissances ordinaires aux savoirs savants sur le langage, les langues et la communication verbale. Ceux-ci sont en principe produits par des spécialistes socialement identifiés comme tels, qui sont structurés en communauté scientifique et discursive. Il serait hasardeux d'en conclure à une hétérogénéité des deux ensembles et donc de les opposer frontalement car, d'une part, les savoirs savants se fondent sur des savoirs ordinaires, au moins celui dit autoreprésentation des langues qui constitue le déjà-là à partir duquel ils sont tenus de constituer leurs concepts et leur démarches. Mais, de l'autre, les connaissances scientifiques sortent des cercles spécialisés et se diffusent dans le grand public, jeune ou adulte, par de nombreux canaux, dont l'éducation scolaire. Ces connaissances savantes, transposées ou "manuelisées » (Collinot et Petiot 1998) peuvent, en conjonction avec d'autres facteurs, modifier ou faire évoluer les représentations sociales dominantes existantes, donner matière à de nouvelles représentations, entrer telles quelles dans les ressources cognitives des acteurs sociaux où elles se substituent alors aux savoirs antérieurement acquis et utilisés socialement.

Nous avons déjà avancé (Beacco 2001) de telles considérations pour la circulation des «savoirs grammaticaux » en didactique des langues; nous y soulignions, en particulier, que la distinction entre savoirs universitaires sans visée pédagogique et descriptions élaborées en vue de leur implication dans l'enseignement (par le biais d'instruments de référence destinés à l'enseignement / apprentissage ou à l'autoformation) est récente et datable de l'instauration la linguistique comme discipline universitaire (à compter du début des années 70 en France). Avant cette période, l'histoire de la « grammaire » oscille, de manière complexe, entre ces deux pôles (Chevalier et Delesalle 1986). Mais, de fait, les enseignements de la langue comme matière demeurent irrigués par des savoirs savants. Proposés aux apprenants dès le plus jeune âge, ils rendent problématique l'identification de savoirs sur la langue authentiques et premiers, c'est-à-dire non «contaminés » par les con- 
naissances scientifiques pédagogisées. Qui ne dispose pas d'une image du participe passé français et de ses accords? La diffusion des connaissances linguistiques scientifiques est aussi constituée par des discours d'expertise, produits par les spécialistes impliqués dans l'enseignement de la langue de scolarisation. Les débats relatifs à l'enseignement du français ont généralement large audience dans les médias de masse et ils constituent donc une autre opportunité de diffusion de connaissances savantes.

Ces formes multiples de circulation, de contact et d'hybridation des savoirs des linguistes et de ceux des non linguistes rendent délicate la fixation de frontières tout autant que l'identification de connaissances ordinaires, spontanées ou intuitives, qui ne devraient rien aux savoirs savants dans le domaine particulier des langues.

\section{Une entrée méthodologique pour décrire un «idéal discursif ordinaire »}

Ce qui vient d'être exposé nous a conduit à concevoir une démarche spécifique, non nécessairement généralisable, pour aborder une des questions relevant de la linguistique ordinaire. Nous nous proposons d'analyser une représentation sociale non dominante mais que nous considérons comme diffuse (que nous nommerons désormais celle " des gens »), attachée à la notion de bien parler / écrire non scolaire / non normatif. Cela suppose :

- d'identifier des données qui correspondent à l'assise sociale transversale de celle-ci ;

- d'analyser celle-ci non à partir de ce qui pourrait en être dit dans des entretiens sollicités auprès des acteurs sociaux concernés mais, indirectement, à partir de caractéristiques de textes qui entreraient dans la catégorie des formes discursives correspondant à ceux que «les gens » reconnaissent en général comme pour exemplaires de leur «idéal » du bien parler, même si ce canon est peu légitime ou tenu pour marginal ;

- d'essayer de repérer dans le lexique de l'autoreprésentation du français des termes qui pourraient correspondre à ces pratiques communicatives valorisées et dont la glose lexicographique serait en mesure d'enrichir notre appréhension de ces valeurs.

\subsection{L'Équipe, un journal « des gens »}

La première décision, cruciale, concerne le choix des observables. Nous avons décidé de constituer un corpus de textes écrits à partir du quotidien sportif L'Équipe. Ce quotidien national français est le seul à viser le lectorat de tous ceux qui s'intéressent aux événements sportifs et il est sans doute inutile ici de caractériser l'audience médiatique considérable du sport (en particulier télévisuelle), qui a suscité la création de nombreuses chaînes thématiques. De plus son tirage s'établit à des niveaux élevés : en 2007, 336.929 exemplaires (diffusion totale payée) ${ }^{(2)}$, ce qui le situe au quatrième rang des quotidiens nationaux (adhérents à l'ODJ), derrière Le Parisien + Aujourd'hui en France (534.032), Le Monde (358.655), non loin du Figaro (344.479). Mais en nombre de lecteurs L'Équipe

(2) Source ODJ : http :// www.odj.com, consultée le 25 mai 2008. 
vient en tête (2,3 millions), devant Le Monde (2,03 millions), selon des résultats de l'enquête 2007 Audipresse-EPIQ.

Outre cette audience quantitative qui en fait certainement un quotidien « des gens », le lectorat de L'Équipe est trans-groupe : les données de 1962 (citées par Seidler 1964) font ressortir que le lecteur de L'Équipe est plus masculin, plus jeune, plus citadin et surtout plus instruit et d'un niveau social et économique plus élevé par rapport à l'ensemble de la population. Selon les données 1974 du Centre d'étude de support de publicité (CESP ; citées par Derieux et Texier), L'Équipe est surtout lue par les cadres moyens $(25 \%)$ et les contremaîtres et ouvriers qualifiés (21\%), mais les employés (12\%) les ouvriers spécialisés et manœuvres (11\%) et les cadres supérieurs $(10 \%)$ sont présents de manière significative et assurent ainsi la mixité sociale et socioculturelle des lecteurs. Sa pénétration dans les catégories socioprofessionnelles dites + (cadres, professions libérales, petits patrons et professions intermédiaires) ne se dément pas : son audience (en milliers de lecteurs au numéro moyen) est de 749, alors qu'elle est de 907 pour Le Monde, 765 pour 20 Minutes (données Audipress-EPIQ 2007).

Cet ensemble de données sociologiques semble assurer que L'Équipe est un " journal populaire » au sens où sa diffusion est large mais, en même temps, non centrée sur un groupe social particulier.

\subsection{Lire L'Équipe, une pratique culturelle peu légitimée}

Or, malgré l'amplitude notable de son audience, qui en fait un quotidien de type « majoritaire », la lecture de L'Équipe est perçue comme un trait de comportement culturel peu légitime au regard des normes socioculturelles dominantes.

Dans l'analyse qu'il consacre à l'enquête de l'Observatoire national de la vie étudiante (OVE), B. Lahire (2002-2004) remarque (note 16 p. 18) que la presse sportive est créditée d'une faible légitimité culturelle, puisque « plus on avance dans le cursus et moins on lit la presse sportive, passant progressivement de $26,8 \%$ de lecteurs à $\mathrm{Bac}+1$ à $11,8 \%$ à $\mathrm{Bac}+6$; ce sont les bacheliers STI $(53,4 \%)$ qui constituent les plus grands lecteurs de cette presse ». Il souligne plus généralement que «à considérer les différences culturelles en matière de lecture de la presse, tout se passe comme si on voyait se recomposer l'espace social dans son ensemble avec un pôle d'étudiants (STS et d'IUT production, étudiants de formation scientifique et technique) caractérisés par les goûts les plus "populaires" (quotidiens régionaux et sportifs) et un pôle étudiants (Prépas Lettres, Droit et sciences économiques, Lettres et sciences humaines) qui ont les goûts les plus légitimes culturellement (quotidiens nationaux et étrangers)» (p. 19). Cette dichotomie très englobante ne tient pas compte, pour ce qui est de la presse sportive, des données de diffusion de L'Équipe rapportées plus haut, ceci du fait de la nature de l'échantillon considéré (les étudiants). Et surtout elle classe a priori la presse sportive et la presse régionale, dont les finalités informatives sont extrêmement différentes, comme marqueur de culture non distinguée.

Mais B. Lahire fait aussi état du fait que « la propension à lire la presse sportive diminue lorsque s'élève le degré de reconnaissance scolaire, passant de 22,8\% de lecteurs (mention Passable [au bac]) à 12,4\% (mention Très bien) » (p. 19). Cette observation est capitale pour notre propos, puisqu' elle donne corps à l'hypothèse selon laquelle la fréquentation de publications comme $L$ 'Équipe est à mettre en relation avec la culture scolaire. Cette relation tendrait à démontrer que l'intégration à la culture scolaire, qui se manifeste par la conformité des résultats obtenus aux 
épreuves (du baccalauréat) c'est-à-dire aux attentes de 1'Institution (qui constitue l'un des critères non explicites d'évaluation des acquis) pourrait comporter des effets sur les représentations discursives, en ce que les normes scolaires d'écriture valorisent certaines formes textuelles au détriment d'autres, dont les textes de la presse sportive feraient partie. Si cela est, un corpus de textes de L'Équipe peut être considéré comme adéquat à notre projet.

\subsection{Lire L'Équipe pour son « style »?}

Reste cependant à démontrer que l'audience trans-groupe des textes de L'Équipe tient aussi, au moins en partie, à l'attrait de leur écriture, laquelle serait alors caractérisable par des réalisations linguistiques valorisées par le lectorat « des gens ». Ces traits d'écriture, dits communément style, sur lesquels prennent appui des jugements positifs rapportés aux valeurs d'une esthétique de masse partagée, pourraient ainsi constituer une actualisation des représentations sociales de ce qu'est le «bien écrire » ordinaire, qui contrevient au modèle discursif scolaire, contraignant mais socialement distinguant.

La première observation que l'on peut avancer à cet égard est que les textes de L'Équipe qui relatent, pour une partie d'entre eux, des rencontres ou événements sportifs ne répondent que d'assez loin au cahier des charges professionnel des journalistes. Si «la-politique» en est effectivement exclue, en conformité avec le code déontologique du quotidien, on ne voit pas comment ces textes répondent aux objectifs habituels de la presse écrite :

- « rapporter de façon concise », car un match devrait alors se résumer à son résultat, accompagné d'un bref commentaire, à la manière des informations télévisuelles (qui donnent tous les résultats de Ligue 1, par exemple) ou des brèves sportives des quotidiens nationaux généralistes;

- «permettre au lecteur de se faire son propre jugement», mais le lecteur a sans doute vu le match dont il lit le compte-rendu et il a souvent des préférences partisanes lui-même, en tant que supporter, ce qui le conduira éventuellement à approuver ou contester plus ou moins vivement le point de vue du journaliste ;

- « séparer l'information du commentaire », ce que ne font franchement pas des titres comme «Scénario catastrophe», (Une, 8 sept. 2007) ou «Une fin pitoyable » (Une, 20 oct. 2007) et « Une totale faillite » (même jour, p. 25)

La simplicité devrait y être de mise pour éviter des efforts aux lecteurs : ne pas employer de mots rares, de tournures recherchées, bannir les subordonnées. Mais que dire de : «Les munitions, les Français les ont eues. Suffisamment pour se mordre leurs doigts gourds ou s'arracher les yeux qu'ils n'ont pas eus en face des trous pour éviter les interceptions $"{ }^{(3)} 8$ septembre p. 25), où figurent une locution-valise et deux défigements. La précision y est cependant au rendez-vous, puisque la terminologie technique s'impose (« une passe lumineuse de l'intérieur du pied », avec un appréciatif peu technique, cependant ; 22 sept. 2007 p. 19).

Reste l'exigence de vivacité, de « vivant», destinée à «capter l'attention »du lecteur. Elle devrait se matérialiser par 1'emploi de la première personne (rare dans notre corpus), par l'emploi du présent (mais l'alternance des temps est de règle dans le corpus), par des citations (inexistantes dehors des interviews) et des

(3) Exemple choisi au hasard. 
images ou comparaisons, comme le recommandent les manuels de technique du journalisme. Or nous verrons que, s'il semble bien que ce soit la vivacité qui domine ces textes, elle s'accommode facilement de la complexité. Cette vivacité d'écriture est effectivement largement fondée, on le montrera en 3, sur ce qu'on peut nommer, en première approximation, des « images et comparaisons », et dont on peut estimer, en fait, qu'elle réside dans une certaine forme de connivence scripteur-lecteur partageant comme une « joie de dire » qui alimente celle de lire.

En second lieu, il importe de relever que la nature même de la communication sportive écrite implique de faire porter l'accent sur l'écriture : ces textes sont le plus souvent « du lendemain » et ils s'adressent à des lecteurs qui sont aussi des spectateurs / téléspectateurs et qui ont donc, dans bien des cas, suivi en direct, des stades ou de leur fauteuil, les épreuves et rencontres dont ils retrouvent une représentation écrite dans leur quotidien. La fonction informative est y passablement faible pour les grands événements sportifs ou pour ceux qui se sont déroulés dans l'environnement local ou régional des lecteurs. De plus, les scénarios des rencontres sportives sont infinis, mais ils procèdent d'un répertoire fourni mais fini d'éléments constitutifs. Par exemple, pour le football, ce sont des «événements » comme : but à dernière minute, erreur d'arbitrage, expulsion, jeu lent, manque de réussite des avants, mésentente des arrières, état du terrain, réactions du public... C'est à partir de cet inventaire d'éléments narratifs que se construira, dans l'écriture, la trame sémiotique du texte. C'est dire que celle-ci sera peu centrée sur l'information puisque cette dernière réside essentiellement dans la combinatoire et non dans l'apport de connaissances inédites.

Guettés par une répétitivité structurelle, peu pertinents en termes strictement informatifs sinon dans les détails, on conçoit que les textes de L'Équipe s'en remettent volontiers à la perspicacité technique et analytique du commentateur tout autant qu'à la vivacité de sa plume, tout comme l'on attend des journalistes sportifs du direct la précision technique du commentaire associée à la passion : chaleur émotive et enthousiasme communicatif.

Il nous semble qu'à ces titres $L$ 'Équipe peut bien être analysée dans son écriture en tant que celle-ci laisse affleurer des traits d'un bien écrire apprécié par « les gens $»$.

\section{Des traits d'un idéal d'écriture ordinaire}

Le corpus constitué dans L'Équipe l'a été pour éviter d'accentuer outre mesure les effets de variété dus à la diversité des sports, à l'importance des événements sportifs... On a choisi l'ensemble des textes concernant la Coupe du monde de rugby 2007, présentés dans le cahier spécial du journal du 8 septembre au 22 octobre. Compte tenu de la taille du corpus (une quarantaine de fascicules de 8 à 10 pages), on ne présentera ici que des sondages exploratoires qui demandent à être vérifiés ${ }^{(4)}$. Et les résultats de l'analyse pourront être faussés par les catégories analytiques retenues, d'autant que toutes les catégories ordinairement employées en analyse du discours n’ont pas été utilisées dans la présente analyse.

(4) La référence aux textes (tous de 2007) sera donnée sous la forme : date (de publication figurant sur le quotidien)/ numéro de page ; par exemple : 11 sept. / 15. 


\subsection{Les titres}

Élément d'accroche par excellence, les titres à la une, dans une tradition éditoriale bien établie pour L'Équipe, ne sont jamais directement informatifs / descriptifs. Ils peuvent y tendre comme : «Le Sud trace sa route» (24 sept. / 21) ou « Les Blacks éclipsent les Bleus» (9 sept. / 17) mais ils sont surtout allusifs ( Pour choisir son destin », 10 sept. / 19). La modalité appréciative, dans des valeurs affectivisées extrêmes, est souvent sollicitée : par exemple, «Le grand gâchis » (15 oct. / 1) ou « Une fin pitoyable (20 oct. / 1) font pendant à «C'est immense » (7 oct. / 1) de quelques jours plus tôt. Ces recours à l'appréciatif ne sont pas caractéristiques de l'Équipe, car ils sont volontiers utilisés dans la presse à scandale, la presse people et dans d'autres supports.

Plus caractéristiques, des titres dont les destinataires sont les sportifs eux-mêmes, lesquels ne sont cependant pas actualisés linguistiquement, sous forme d'adresse. On a relevé comme exemples de ces incitations et exhortations : « Le jeu, rien que le jeu » (11sept. / 15), avec une construction détachée et une reprise littérale qui donnent une dimension d'oralité au syntagme ; ou, plus explicite : « Il va falloir les ${ }^{(5)}$ suivre » (15 sept. / 21), avec un cataphorique (Ils = l'Afrique du Sud) dans le chapeau qui suit, et un déontique, explicite cette fois.

Les titres de L'Équipe semblent être formés avec une certaine prédilection sur des échos intertextuels : «À pied à cheval ou en avion » (25 sept. / 15), détournement de «À pied, à cheval ou en voiture » (titre d'un film français de 1957, avec Noël-Noël et Darry Cowl), « 15 hommes en colère » (27sept. / 17), où l'on aura reconnu le titre du film de S. Lumet ( long dimanche de rugby » (30 sept. / 1), autre film mais de 2004 («Un long dimanche de fiançailles »), et sans doute plus présent dans les mémoires. Ces variations sur des titres, citations... sont bien attestées dans la publicité et dans la presse françaises, mais il est significatif, que fortement sollicitées par Le Nouvel Observateur ou par Libération, elles le soient aussi dans un quotidien tenu pour populaire.

Est enfin présent la technique du « jeu de mots », substitution d'hétérographes homophones, dont Le Canard enchaîné s'est fait une spécialité, comme dans «Il était une foi » (2 oct. / 23) ou dans «Le non de la Rose» (14 oct. / 1), qui met aussi en jeu un détournement intertextuel (reprise du titre du roman (1980) de U. Eco, «Il nome della rosa »).

Cette diversité de procédés répond à de fortes nécessités éditoriales et on y utilise donc la totalité des formes d'écriture dont l'expérience de journaliste montre qu'elles pourraient « accrocher» le lecteur. En ce sens, les titres donnent un avantgoût de l'écriture des textes qu'elle met ainsi en exergue. Il est significatif qu'ils puisent une partie des procédés qui les constituent dans ce que l'on pourrait nommer des jeux sur la langue et sur le discours, où vient se concrétiser une certaine forme de créativité verbale.

\subsection{La structure des articles}

Les articles de l'Équipe, comme dans bien d'autres genres discursifs, ne présentent pas d'indications métalinguistiques (annonce du plan...) et leur structure tient davantage de l'allure " à sauts et à gambades », chère à Montaigne, que du classique exposé à orientation argumentative en trois points. L'objet du reportage d'un

(5) = l'équipe d'Afrique du Sud. 
match est en principe son récit (qui comprendra des éléments descriptifs) et son commentaire. Or, dans ces textes de l'Équipe, le récit n'est pas chronologique / linéaire et le commentaire n'est pas distingué spatialement du récit et confiné dans des paragraphes identifiés comme tels; au contraire il sature tout le texte. On le montrera à partir de deux exemples.

Dans un article comme «Un mur bleu et deux éclairs » (7 oct. / 18), le récit est fortement désarticulé, comme émietté au milieu d'analyses explicatives : le $\S 1$ explique les raisons de la victoire de l'équipe de France sur les All Blacks de manière générique, en analysant la stratégie, sans renvoi à des moments précis du match (« en les écœurant en défense, en les dominant au combat collectif, en les faisant déjouer... $\left.{ }^{(6)}\right)$. Le $\S 2$ relève du bilan global, mais effectué à la modalité appréciative («Quel paradoxe ! » ouvre le $\S$ ) et il s'articule autour de deux constats repérés de part et d'autre d'un articulateur («Et, en sens inverse...»), dont c'est là un des rares emplois dans ce texte, hors de la séquence narrative. Les paragraphes suivants analysent la défense des Français et le coaching (considéré comme capital pour « l'essai de la gagne », marqué à la $69^{\mathrm{e}}$ minute). C'est immédiatement après ce premier renvoi circonstancié à l'historique du match, soit aux deux tiers de l'article, que se déploie une séquence narrative, toujours appréciative, dont rien ne signe le début et surtout pas le contenu l'intertitre («Le pied pour le pied») qui en scande visuellement l'amorce. Celui-ci se constitue sur un fond initial (qui n'est cependant pas une situation de départ : "La tactique n'avait pas fait de mystère ») et s'organise autour de verbes d'action, tous au... passé simple, dont la disparition a peut-être été annoncée un peu prématurément (« les Bleus passèrent l'essentiel de la première période... ils ne se découvrirent pas... Williams fut poussé en touche... »). Ces deux paragraphes à régime narratif sont balisés par un réseau de repères inhabituellement dense, qui marque les moments forts de la rencontre (« Mais ils ne se découvrirent pas... On crut bien cependant que... Une première fois... Puis... Mais la rectification arriva... »). Le récit se clôt par un regret (« Dommage... »), par un hymne à la mêlée française, dont il emprunte littéralement les accents ( "Gloire soit rendue à...»). La chute est, elle, en forme de maxime d'allure analogique : "La mêlée, c'est le baromètre de l'âme d'une équipe et l'équipe de France avait hier soir une belle âme ».

Un article comparable, situé en page 2 du Cahier Coupe du monde, rend compte de la défaite de l'équipe de France devant l'Angleterre (14 oct. / 18). On y retrouve sans peine la même désinvolture de planification, que l'École sanctionnerait sans doute vertement. L'attaque ( $\$ 1)$, in medias res comme il convient, énonce 1'isotopie dominante qui structurera le commentaire du scripteur (« ...fil du rasoir...»). S'y enchaîne (§ 2), sans délimitation discursive, la représentation de la première mi-temps, qui est descriptive / globale et non événementielle (« la qualité des deux défenses... la promptitude à exploiter toute petite faute...»). Il faut attendre la fin de ce paragraphe pour que soit mentionnées des phases de jeu précises ( $448^{\mathrm{e}} \mathrm{mi}$ nute... $74^{\mathrm{e}}$ minute $\left.»\right)$. Le récit, toujours au passé simple, prend une allure linéaire plus attendue ensuite ( $§ 3$ à $\S 5)$, en mettant en relief des événements spécifiques, ce qui n'empêche pas des retours de la description englobante peu chronologisée ( La défense blanche repoussa avec vigueur les assauts...») ou l'insertion d'énoncés évaluatifs entiers comme : «Les Français pourront avoir des regrets limités. Ils n’ont pas fait assez pour... ». Le $\S 6$ continue sur la lancée globaliste, avec des hyperonymes permettant de classifier des phases de jeu (« ...bataille des avants..., les

(6) Noter, au passage, l'emploi néologique. 
regroupements...»), alors que la fin du texte revient sur la première période, mêlant description stratégique, c'est-à-dire abstraite par rapport à l'observable ( «...l'accent était mis sur le jeu au pied...»), et narration («l'essai de la deuxième minute dans Anglais....). La chute est en forme de morale évangélique, détournée par un à peu près phonétique («Ayant choisi de vivre par le pied, la France finit par périr par le pied, majestueux il est vrai, de Wilkinson »), qui transforme la phase de jeu finale en destin tragique mérité.

Ces trajectoires textuelles improbables caractérisent le présent corpus et bien d'autres textes journalistiques. Elles constituent l'exact contraire des écritures scolaires non créatives (1'écriture créative ${ }^{(7)} \mathrm{n}$ 'ayant fait son entrée que tardivement dans les programmes et les pratiques), qui suppose programmation, organisation textuelle et traces visibles de cette organisation dans des genres discursifs scolaires et universitaires. Pourtant cette forme de déceptivité structurale tend ici à accompagner et soutenir une lecture intégrale, sinon linéaire, des textes, précisément parce qu'elle joue de l'imprévu, semblable en cela aux " règles » de genres d'écrits scolaires pratiqués dans d'autres communautés de communication (le tema italien, par exemple), où la présence de structures de texte affichée est volontiers assimilée à de la rigidité. Ce qui est donc appréhendé non comme une forme de brouillage et de confusion mais comme une forme de souplesse, semble mimer, dans «l'esthétique des gens », telle qu'elle se dessine dans ce corpus, une liberté d'allure et de parole préclassique et redonne valeur à la spontanéité face aux discours policés et à la dispositio linéaire.

\subsection{L’appréciatif}

Nous avons déjà eu l'occasion de noter l'omniprésence des modalisations appréciatives. Elles se rencontrent indifféremment dans tous les éléments constitutifs de ce genre discursif (récit, description, commentaire, analyse, attaque, chute...), au point que l'analyse de ses constantes de distribution s'avère inutile pour caractériser cet ensemble de textes. Restent alors les constantes de représentation, c'est-à-dire les régularités décelables dans l'emploi des moyens verbaux qui servent à l'actualiser.

L'appréciatif est surtout utilisé comme commentaire ou comme doublant les commentaires explicites du journaliste. La fonction informative principale attendue du journaliste est d'évaluer les résultats obtenus par les sportifs, dans le cas présent, la qualité du match et celle du comportement de l'équipe de France, les équipes adversaires étant elles aussi objet de notations évaluatives, mais dans une moindre mesure, cette focalisation des appréciatifs sur l'adversaire étant majeure quand l'équipe de France est perdante.

Le compte-rendu du match d'ouverture France-Argentine (perdu par la France) (8 sept. / 22) comprend, dans son premier paragraphe, des appréciatifs comme : « défaite tactique de grande ampleur (sous-titre), incapable de mettre en place..., a nettement perdu..., résultat catastrophique..., faiblesse dans l'adaptation..., déficiences dans... ». Y alternent des évaluations quantitatives («nettement») et d'autres, d'orientation qualitative, actualisées par des adjectifs ou des noms (négatifs / péjoratifs ici). On notera le soin apporté à varier les ressources verbales utilisées. Le jeu des Argentins est qualifié d' « intelligent» et « parfois très varié ». L'ensem-

(7) Formes de productions scolaires faisant appel aux techniques d'écriture automatique/à contrainte, de type OULIPO, ou d'imagination. 
ble de ces notations vient confluer dans le diagnostic « on ne peut bien entendu pas parler de hasard ou de mauvaise chance ». Dans un autre article (7 sept. / 22), on peut aussi relever la forte densité d'emploi de la modalité appréciative, attachée en particulier à des éléments du jeu, déjà qualifié globalement dans le sous-titre (« débauche de jeu ») : « ...maul mal fagoté...» « ...relâchement en défense...» «...jeu au pied trop faible... », « ...surnombres mal négociés... » et qui peuvent venir en série : les trois premières se trouvent dans la même phrase.

Ce qui frappe est l'extrême diversité des moyens, là où d'autres genres discursifs en sont réduits à un éventail limité qui conduit à des tournures quasi stéréotypées («Il est important de..., il est intéressant de... » dans les discours de recherche). Les All Blacks (9 sept / 17) jouent « un rugby d'école», qu'ils « récitent sans bafouiller », "ils ont honoré leur statut» (de favoris), avec une « démonstration de force et de conviction»; les Sud Africains sont dits évoluer « ...aux mêmes allures. Plus vite, plus fort et plus spectaculairement que... », où l'on relève dans la formulation rhétorique ternaire une rupture de symétrie (deux adjectifs mais un adverbe) qui vient déjouer les anticipations du lecteur. À noter, presque comme exemple de démonstration, un énoncé tel que « Le nombre d'en-avant, d'approximations, de ballons perdus, qui émaillèrent leur sortie contre l'Écosse, l'impression étonnante d'une certaine nonchalance qui caractérisa le match peuvent entretenir cet espoir...» (24 sept. / 21) où la série des sujets du verbe est comme une litanie négative constituée de termes techniques, qui désignent des erreurs dans le jeu, et de termes non techniques, nominaux (《 approximations, nonchalance ») et adjectivaux ( «étonnante»), avec des variations secondaires sur la quantification : pluriel pour « approximations » et « une certaine » pour «nonchalance».

Si la présence d'appréciations, voire leur nombre (surtout élevé dans les articles entre les rencontres), répond aux conditions de production et de réception de cet ensemble de textes, il est notable que leur mise en mots dénote ce souci de diversité. Celui-ci laisse à penser que l'on n'est peut-être pas seulement dans le domaine de la recherche de la formule ou de l'effet, mais dans celui de l'écriture au sens où l'entendait J. Peytard (1982 p. 99) : «Si l'on admet que l'écriture n'est pas ornementation, que le scripteur ne cherche pas à bien dire ou mieux dire par des écarts et trouvailles multiples sur son texte mais qu'il invente le langage, alors l'attente porte sur le signifiant, sur le travail du signifiant». Si la notion de travail ne semble pas pouvoir convenir à l'écriture de L'Équipe, puisqu'elle demeure dans le cadre de la norme langagière et discursive (voir l'utilisation du passé simple qui peut sembler presque hypercorrecte, par rapport à ses emplois limités dans les échanges ordinaires), celle de la rédaction faite de trouvailles ne semble pas non plus y correspondre. Mais il est clair que ces textes pour le plus grand nombre traduisent un intérêt du lectorat pour les formes du signifiant.

\subsection{Locutions et phraséologie}

Cette focalisation sur l'écriture conduit à l'emploi de locutions et d'expressions figées, de maximes ou de formulations quasi proverbiales, dont l'emploi imagé, nouveau (par défigement ou altération) ou archaïsant, tranche sur le langage de la conversation ordinaire. Ce relatif dépaysement lexical, qui met en relief la « richesse » de la langue, dans son historicité, met aussi en avant l'habilité de qui la convoque à bon escient dans des textes où, a priori, elle a peu à faire.

Le recours au locutionnel peut ne pas être saillant, quand il est inséré dans un énoncé, particulièrement quand il ne prend pas une forme phrastique. Dans «...en 
plaçant sur sa route des Anglais, loin de compter sur la même armada qu'en 2003 ...» (15 oct. / 21) l'emploi d'armada n'est pas franchement locutionnel, puisque invinciblen'y figure pas (et d'ailleurs ill'eût été à contresens, puisque l'Invincible Armada a été envoyée en 1588 par Philippe II d'Espagne... contre l'Angleterre). Il l'est manifestement dans : « ...juste le petit poison du doute s'est installé dans les esprits » (6 oct. / 17), avec une variation peut-être non contrôlée par rapport à instiller (poison) ou insinuer (doute).

À côté d'autres emplois simples comme «...un doigt accusateur va bientôt être pointé en direction de...» (28 sept. / 14), on rencontre aussi des formulations complexifiés : pour représenter la difficulté du choix de la sélection contre les All Blacks (3 oct. / 15) on écrit : «ça reste la bouteille à l'encre », qui devient dans le titre : « La bouteille à l'encre noire », adjonction-surcharge qui rappelle la tenue des adversaires.

De l'emploi de locutions disponibles dans le lexique, on passe aisément à des formules qui adoptent une allure de locution, mais qui sont en fait des créations $a d$ hoc: «...ils ont vérifié que les Irlandais avaient encore de la couenne » $(22$ sept. / 19), variation sur « avoir la peau dure »; «La France est sortie par le soupirail de la Coupe du monde » (20 oct. / 17), variation plus réaliste de " sortir par la petite porte » et qui laisse entendre que ladite équipe devrait être remisée à la cave ; « ils ont loupé la sortie et leur ordinateur de bord a dû calculer un itinéraire de secours » (25 sept. / 15). Dans la même veine, sur le modèle des locutions de forme « adjectif comme nom », mais avec une variation (le nom est lui-même déterminé), a été créé « [ce fut] acharné comme des setters d'Irlande sentant la plume» (22 sept. / 19) qui ouvre, on s'en doute, un reportage sur une rencontre France-Irlande. Les comparaisons se font plus amples : «Elissalde s'enfuit en arrière avec le dernier ballon, comme on dérobe le goûter dans la cour de l'école» (7 oct. / 19), entre Homère et la «Guerre des boutons ».

On ne fait pas appel à des proverbes, mais on repère çà et là des maximes, prolongement des sententiae latines, plutôt pittoresques comme, en chute : «Au vu des bruits des derniers jours, c'est même reparti comme au mauvais vieux temps : la grande surface du bricolage est de nouveau ouverte » (20 oct. / 17), qui comporte aussi une locution détournée ( «bon vieux temps») et évoque le bricolage à venir par référence aux magasins de l'enseigne « Monsieur Bricolage». Plus ambitieux mais avec un brillant verbal, fait de jeux de mots en chaîne et de mélange de tons délibérée : «Le rugby est un art poétique brut et même quelquefois brut de brutes » (7 oct. / 9), qui mêle Horace (ou Boileau), l'art brut et, en passant, le mousseux. La maxime vise même à la plénitude philosophique, dont elle mime l'expression paradoxale dans : "Au fond on touche là à l'identité de la France, indéfinissable en temps de paix et cristalline en temps de crise, dans sa férocité à détruire les mythes pour mieux construire le sien » (7 oct. / 19), formulation exceptionnelle à la hauteur l'événement historique qu'elle célèbre (victoire de la France sur les All Blacks), qui ne répugne pas à convoquer F. Braudel ( L'identité de la France ») ou, du moins, le syntagme fortement idéologique que l'historien a retenu comme titre.

La forte présence de locutions et de créations à base de locutions ou qui en adoptent les formes syntaxiques confirment que la capacité des scripteurs à mobiliser des ressources lexicales d'emploi rare ou à créer de la phraséologie, même non destinée à perdurer, constitue un trait d'écriture valorisé par les lecteurs. Il ne s'agit pas là d'une réponse à un besoin, mais d'une recherche d'adéquation entre des formes textuelles attendues et leur réalité linguistique. C'est probablement de cette rencontre que naît le plaisir du texte. 


\section{Idéal discursif et métalangage interne : la "verve» comme créativité}

Les traits d'écriture relevés dans le corpus de L'Équipe ne doivent pas faire oublier que la totalité de ces textes n'est pas uniformément de cette nature et qu'ils adoptent aussi des formes plus communes. Mais leur saillance invite à les considérer comme donnant sa coloration spécifique à ce genre de discours. Comme nous l'avons noté, ces régularités discursives sont à reconduire à des compétences d'écriture valorisées comme :

- la capacité à improviser, à écrire au fil de la plume, à ne pas se contraindre par un plan ou une organisation préétablie ;

- la capacité à mobiliser du lexique très varié et peu usité, pour créer globalement des effets de richesse, qui valorise la langue elle-même, ou d'abondance rhétorique (la copia cicéronienne);

- la capacité à créer des textes « chaleureux » et non énonciativement tenus à distance, par le suremploi de la modalité appréciative ;

- la capacité à jouer sur la langue en s'en éloignant, en la parodiant ou en la détournant, sans souci d'homogénéité de ton (passé simple vs « brut des brutes »), en prenant toutes libertés avec le « code » et les normes sociolinguistiques, mais en demeurant dans le cadre de l'acceptable, c'est-à-dire de la nécessité de faire sens immédiatement.

Jeu, liberté, abondance, affect sont ainsi les composantes d'une forme de créativité langagière et discursive qui est à considérer comme correspondant à une valeur esthétique partagée par le plus grand nombre. Il est inutile de montrer en quoi elle se distingue radicalement des normes scolaires et universitaires françaises, qui sont décrites par M.-A. Paveau et L. Rosier $(2008)^{(8)}$. Cette valeur pourrait correspondre à un terme du métalangage interne : verve Le sens littéraire dominant de ce mot, attesté depuis 1694, est selon le «Trésor de la langue française » ${ }^{(9)}$ :

- inspiration vive et chaleureuse, imagination créatrice (écrire de verve $=$ d'un premier jet, dans l'improvisation) ;

- excitation poussant à faire quelque chose avec fougue, vivacité ;

- qualité brillante d'une personne qui s'exprime oralement ou par écrit, brillant étant défini comme : qui séduit ou frappe vivement l'imagination ou l'esprit.

Cette description lexicographique correspond assez exactement aux traits linguistiques identifiés dans le corpus et ce terme est donc éligible pour les dénommer. D'autres candidats possible comme bagout (bavardage volubile où entrent de la hardiesse, de l'effronterie et l'envie de duper) ou faconde (grande facilité de parole) sont plus restreints et plus éloignés de nos données. Verve, qui s'oppose assez nettement à platitude, désigne ainsi une forme de créativité.

Cette rapide caractérisation de la « verve écrite » trouverait facilement des correspondants dans les pratiques orales. Cette dernière a été décrite, en particulier, dans les interactions orales, à partir d'interrogations sur la créativité verbale. Dans son ouvrage, fondamental à cet égard, R. Carter (2004) analyse, à partir du

(8) Voir le chapitre $7:$ «Le bon style. Les belles manières d'écrire », et en particulier 7.3: Les stigmates de la phraséologie.

(9) Site http ://atilf.atilf.fr consulté le 30 mai 2008. 
corpus oral anglais CANCODE ( 5 millions de mots), des pratiques langagières : "Everyday conversation reveals uses of language that are strongly associated with criteria of 'litterariness' [...] Recent studies also recognise that casual conversation intrinsically creates a space within which speakers can fulfill what would appear to be a fundamental need to insert more personnal or personally evaluative position into the ongoing discourse" (p. 79) ${ }^{(10)}$. Dans la deuxième partie de cette étude ("Forms and fonctions"), il décrit de manière très précise des données conversationnelles qui l'amènent à conclure que "in relation to spoken-language uses in the CANCODE corpus examined [...], the most frequent forms of linguistic creativity include :

- speaker displacement of fixedness, particularly of idioms and formulate phrases ;

- metaphoric extension ;

- morphological inventiveness ;

- verbal play, punning and parody throught overlapling forms and meanings ;

- 'echoing' by repetition, incluing echoing by means of allusion and phonological echoes” (p. 109) ${ }^{(11)}$.

On notera, en particulier, le premier élément de cette liste qui s'accorde tout à fait à ce que nous avons relevé dans le corpus L'Équipe (voir 3.4). Et, en conclusion de ce très stimulant ouvrage, R. Carter pose la question de savoir si, plus fondamentalement encore, le problème ne serait pas de se demander "what does a study of spoken language tell us about creativity" mais bien "what does the study of spoken creativity tell us about the nature of language" (p. 215) ${ }^{(12)}$.

Cette étude de la verve, représentation sociale diffuse mais non légitime, a conduit des données linguistiques à la confrontation avec le métalangage interne. On a montré comment celui-ci agit sur les comportements discursifs en tant que constituant un « horizon d'attente » qui informe et surdétermine les productions verbales. Il prend une réalité langagière perceptible qui remotive et relégitime, en principe, la valeur discursive dont il procède. Il serait sans doute opportun d'explorer d'autres valeurs discursives ordinaires... Et de mettre ces «valeurs discursives « des gens » en relation, comme le suggère R. Carter, avec des textes catégorisés comme littéraires ou considérés comme non littéraires mais légitimes, qui relè-

(10) «La conversation quotidienne révèle des usages du langage qui sont fortement associés aux critères définissant la littérarité [...] Des études récentes montrent aussi que la conversation ordinaire crée intrinsèquement un espace que les locuteurs peuvent investir au moyen de ce qui peut apparaître comme une exigence fondamentale d'insérer une position plus personnelle ou relevant d'une évaluation plus personnelle dans le discours en cours » (notre traduction).

(11) «En relation aux usages du langage parlé de CANCODE [...] les formes les plus fréquentes de créativité comprennent :

- les déplacements des formulations fixées opérés par le locuteur, en particulier ceux des expressions idiomatiques et des énoncés formulaires ;

- les extensions métaphoriques;

- l'inventivité morphologique ;

- les jeux de langage, les jeux de mots et la parodie, au moyen de superpositions de formes et de significations ;

- des « effets d'écho », y compris les échos par allusion et les échos phonologiques » (notre traduction).

(12) «...de se demander ce qu'une étude de la créativité orale nous apprend de la langue parlée mais bien ce qu'une étude de la créativité orale nous apprend sur la nature du langage » (notre traduction). 
vent d'une esthétique de la verve, ceux, par exemple (issus de notre panthéon personnel) de Rabelais, de Montaigne, du La Fontaine des Fables, de G. Brassens ou du « Billet » du Monde.

\section{Références bibliographiques}

BEACCO, J.-C. (1982) : La rhétorique de l'historien, Berne, P. Lang.

- (2000): «Les communautés discursives », dans Beacco J.-C. (dir.), L'astronomie dans les médias, Paris, Presses de la Sorbonne nouvelle, pp. 10-23. - (2001) : "Représentations métalinguistiques ordinaires et enseignement / apprentissage de langues », dans Bouquet S. (dir.), Théories linguistiques et enseignement du français aux non francophones, Le français dans le monde, Recherches et applications, $\mathrm{n}^{\circ}$ de juillet (58-80).

- (dir.) (2004) : "Représentations métalinguistiques ordinaires et discours », Langages 154 .

BeAcco, J.-C., Bouquet, S. et Porquier, R. (1999) : Niveau B2 pour le français, Paris, Didier.

BEAcCO, J.-C. et Petit, G. (2004) : «Le lexique ordinaire des noms du dire et les genres discursifs », Langages 154, pp. 85-100.

CARTER, R. (2004) : Language and creativity. The art of common talk, Londres et New-York, Routledge.

Chevalier, J.-C. et Delesalle, S. (1986) : La linguistique, la grammaire et l'école, 1750-1914, Paris, A. Colin.

Collinot, A. et Petiot, G. (dir.) (1998) : «Manuélisation d'une théorie linguistique : le cas de l'énonciation », les Carnets du CEDISCOR 5.

DERIEUX, E. et TEXIER, J.-C. (1974) : La presse quotidienne française, Paris, A. Colin.

GADET, F. (1989) : Le français ordinaire, Paris, A. Colin. - (2003) : La variation sociale en français, Paris, Ophrys.

GESTER, F. W. (2001) : «On relationship between lay interests in language, popular linguistic beliefs and attitudes, and 20 th century linguistics », dans Kniffa H. (ed.), Indigenous Grammar Across Cultures, Berne, P. Lang.

Houdebine, A.-M. (2002) : L'imaginaire linguistique, Paris, l'Harmattan.

JODELET, D. (dir.) (1989) : Les représentations sociales, Paris, Presses universitaires de France.

LAHIRE, B. (2002-2004) : «Formes de la lecture étudiante et catégories scolaires de l'entendement lectoral », Sociétés contemporaines 48, pp. 87-107.

MARTINS-BAltAR, M. (1976) : «Actes de parole », dans Coste D. et autres, Un Niveau-Seuil, Hatier et Didier, Paris, pp. 83-224.

Oger, C. et Ollivier-YANiv, C. (2007) : «Analyse du discours et sociologie compréhensive. Retour critique sur une pratique de recherche disciplinaire », dans Bonnafous, S. et Temmar, M. (dir.), Analyse du discours et sciences humaines et sociales, Gap-Paris, Ophrys, pp. 39-51. 
Paveau, M.-A. et Rosier, L. (2008) : La langue française. Passions et polémiques, Paris, Vuibert.

PEYTARD, J. (1982) : «Sémiologie du texte littéraire et didactique du FLE », Études de linguistique appliquée 45, pp. 91-103.

PY, B. (2004) : "Pour une approche linguistique des représentations sociales », Langages 154, pp. 6-19.

SEIDLER, E. (1964) : Le sport et la presse, Paris, A. Colin.

VIVÈS, R. (1998) : «Les mots pour le DIRE : vers la constitution d'une classe de prédicats », Langages 131, pp. 64-76.

- (2004) : "Les verbes de parole», dans Beacco J-C. (dir.), Niveau B2 pour le français. Textes et références, Paris, Didier, pp. 203-238.

WIERSBICKA, A. (1985) : «La quête de primitifs sémantiques », Langue française 98, pp. 9-23.

\section{ANNEXE}

\section{Un mur bleu et deux éclairs}

C'est d'abord en défense que les Tricolores ont gagné le droit de poursuivre leur parcours. Ils y ont ajouté deux coups de French flair.

\section{CARDIFF - de notre envoyé spécial}

C'EST ENCORE plus grand que la demi-finale de Twickenham (43-31). Cette fois, les All Blacks savaient, ils s'étaient préparés comme jamais, ils en avaient fait une obsession nationale. Et vingt-deux Français remontés comme des coucous les ont battus en les écœurant en défense, en les dominant sur le combat collectif, en les faisant déjouer, en les manœuvrant sur le plan tactique, les forçant à jouer contre nature. Cette Coupe du monde est partie pour ne pas faire exception à la règle : la meilleure défense ne sera pas loin du bout. II faut bien entendu y ajouter la capacité à sauter sur toutes les occasions qui se présentent. Les Tricolores ont réussi leur coup à deux lames. Faire déjouer les Blacks, les déboussoler, rester dans le match au score et choisir le bon moment pour porter le coup de grâce.

Quel paradoxe : voir l'équipe de France, partie de son propre camp, marquer deux essais aux Blacks, I'un sur un renvoi aux 22 mètres vite joué, l'autre sur une mêlée à soixante mètres de la ligne néo-zélandaise, deux bijoux de jeu à la française, celui qu'on croit toujours perdu et qui revient quand on s'y attend le moins. Et, en sens inverse, obliger les All Blacks à tenter de se sortir de la nasse par un jeu de pick and go digne des pires de l'hémisphère Nord, allant de petit tas en petit tas, en faisant craquer une seule fois de cette façon la défense tricolore. Pris à la gorge, les Néo-Zélandais manquèrent cruellement d'imagination, n'utilisant notamment que très peu le petit jeu au pied face à une défense montant pourtant souvent sur un seul rideau.

C'ÉTAIENT LES TRANCHÉES. - En limitant les Blacks à dix-huit points, les Tricolores ont confirmé la qualité de leur défense, probablement la plus efficace de la Coupe du monde. Ils eurent un soupçon de réussite sur deux ou trois franchissements où le joueur néozélandais fut repris par le bout du pied, mais en montant une garde sévère autour des zones de regroupement et en plaquant haut et souvent à deux, ils empêchèrent le jeu néo-zélandais de se développer. Pas de passes sur des joueurs lancés, même quand le premier rideau était 
franchi, pas de possibilité de rebondir sur les extérieurs, pas de mise en situation idéale pour la troisième ligne black, obligée de se coltiner les tâches ingrates. Le jeu néo-zélandais, faute d'une mêlée et de regroupements dominants, était inefficace. Les Tricolores ont plaqué presque deux cents fois, contre moins de cinquante pour leurs adversaires. La stat dit tout...

L'IMPORTANCE DES JOKERS. - Le coaching est souvent surévalué. Pas cette fois. Le coaching des Français a été capital, permettant à la mêlée de tenir le choc, d'amener de la fraîcheur au plaquage avec Szarzewski, Chabal et Poux, et des jambes juste au bon moment avec Michalak, réussissant une course décisive pour lancer Jauzion à l'essai de la gagne (69e).

LE PIED POUR LE PIED. - La tactique n'avait pas fait de mystère. En relançant sans arrêt au pied les ballons et en tentant d'assurer un pressing suffisamment soutenu à le retombée, les Bleus passèrent l'essentiel de la première période sans construire grand chose, attendant une faute néo-zélandaise qui ne venait pas. Mais ils ne se découvrirent pas non plus, entraînant leurs adversaires dans un jeu de gagne terrain qui ne leur convenait pas spécialement. On crut bien cependant que les All Blacks avaient trouvé la solution quand, au quart d'heure de jeu, ils mirent le pied sur le champignon et tentèrent de trouver la faille en commençant à se passer le ballon sur la largeur. Une première fois, Ali Williams fut poussé en touche d'extrême justesse. Puis c'est en plein cœur de l'ouvrage que McAlister trouva deux fois la faille. Il profita là d'un moment de flottement dans la réorganisation défensive qui suivit la sortie de Betsen. Harinordoquy n'a pas le même profil bien évidemment. Mais la rectification arriva assez vite, et plus jamais la défense française ne céda en son cœur.

LA TOUCHE CONTRÉE. - La mauvaise surprise, côté français, vint de là. Alors que la rentrée de Harinordoquy très tôt dans le match leur offrait beaucoup de solutions, les Tricolores se firent contrer trois fois en première période, dont une fois sur une touche importante puisqu'elle permit la première percée de McAlister sur une défense qui n'était pas en place, puis deux fois en seconde période. En fait, les All Blacks qui, dans un premier temps, n'avaient pas tenté de contrer, surent décrypter par Robinson, à la limite de la faute il est vrai, les annonces des Tricolores. Cela ne s'arrangea pas par la suite et un nouveau ballon perdu fut à l'origine de la séquence de jeu qui amena l'essai du break de So'oialo à la 63e minute. Dommage, car cela mit les Bleus sous pression de manière inopportune.

UNE MÊLÉE RETROUVÉE. - Lorsqu'on se souvient de la terrible humiliation de Lyon (473 ) en novembre dernier, la résurrection de la mêlée française, parfois chahutée mais jamais totalement basculée, doit être soulignée. Gloire soit rendue notamment à Pieter De Villiers, énorme en défense et énorme dans l'engagement. La mêlée, c'est le baromètre de l'âme d'une équipe, et l'équipe de France avait hier soir une belle âme.

HENRI BRU

(C) L'Equipe, Cahier spécial Coupe du monde de rugby, 7 octobre 2007 (reproduit avec l'aimable autorisation de L'Equipe) 